\title{
Collective coordinate analysis for double sine-Gordon model
}

\author{
Samira Nazifkar* and Kurosh Javidan ${ }^{\dagger}$ \\ Department of physics, Ferdowsi university of Mashhad \\ 91775-1436 Mashhad Iran \\ (Received on 6 December, 2009)
}

\begin{abstract}
Collective coordinate analysis for adding a space dependent potential to the double sine-Gordon model is presented. Interaction of solitons with a delta function potential barrier and also delta function potential well is investigated. Most of the features of interaction are derived analytically. We will find that the behaviour of a solitonic solution is like a point particle which moves under the influence of a complicated effective potential. The effective potential is a function of the field initial conditions and also parameters of added external potential.
\end{abstract}

Keywords: Topological solitons; Double sine-Gordon; Collective coordinate

\section{INTRODUCTION}

Topological solitons are important objects in various areas of physics and mathematics. Solitons widely appear in nonlinear classical field theories as stable, particle-like objects, with finite mass and smooth structures. They are localized in space as the energy density of these objects is nonzero only in a finite region; i.e. it is significantly nonzero in a small region and goes to zero, exponentially or as an inverse power, as one moves away from this region. They are stable due to nontrivial topological properties of the vacuum manifold. These coherent nonperturbative excitations are distinct from the perturbative excitation objects which correspond to localized small field oscillations around the vacuum $[1,2]$. Solitonic picture of baryons in high energy physics and also Skyrme solitons in nuclear physics are examples of particlelike bahaviour of topological solitons [3, 4] Finding suitable methods for the point representation of soliton solutions of the hierarchies of non-linear evolution equations is an interesting subject in nonlinear field theory (for example see [5]). In this paper we will deal with on of these models.

The sine-Gordon model is probably the most studied integrable model. This model describes a large variety of physical systems ranging from the Josephson effects, particle physics, information transport in microtubules [6], nonlinear optics [7], crystal dislocations [8], and ferromagnets [9]. Double sine-Gordon (DSG) model arises as a sine-Gordon type field theory bearing solitonic solutions with important applications and also attractive mathematical properties. DSG model has been used in describing a multibaryon system. So their solutions appear in the multiflaver spectrum and some resonances in $Q C D_{2}[10]$. There is a very near Relation between DSG model and deformed quantum AshkinTeller model which describes quantum Ising spin chain system [11]. Interacting pair of resistively shunted Josephson junctions and fluxon dynamics in long Josephson junctions are modeled using a DSG formulation [12-14]. Quantum noise of ferromagnetic $\pi$-Bloch Domain Walls also describes using a model with DSG theory [15].

In the inhomogeneous version of the sine-Gordon model, the coefficient in front of the potential becomes a function of space. Recently, there has been an increasing interest in

\footnotetext{
*Electronic address: sa_na262@stu-mail.um.ac.ir

$\dagger$ Electronic address: javidan@um.ac.ir
}

the scattering of solitons from defects or impurities, which generally come from medium properties. It is very interesting to examine the methods of adding the potential to the model on the DSG as a non-integrable model.

Interaction of solitons with defects mainly investigates using numerical analysis and numerical simulations. Some analytical models have been presented which are constructed with using suitable collective coordinate variables. Collective coordinate analysis for point representation of solitons of single sine-Gordon model has been studied before $[16,17]$. In this paper collective coordinate system for DSG model is presented for the first time. The results are more interesting than the single sine-Gordon model, because of very attractive shape of the field potential of DSG model. This system is constructed base on the method of adding the defects by making some parameters of the equation of motion to be function of space.

\section{SOLITONS OF DOUBLE SINE-GORDON EQUATION}

Lagrangian of the double sine-Gordon model in $(1+1)$ dimensions is defined by

$$
\mathcal{L}=\frac{1}{2} \partial_{\mu} \phi \partial^{\mu} \phi-\lambda(2-\cos \phi-\cos 2 \phi)
$$

where $\mu=0,1$. The field equation of motion from the Lagrangian (1) is:

$$
\partial_{\mu} \partial^{\mu} \phi+\lambda(\sin \phi+2 \sin 2 \phi)=0
$$

One soliton solution for the DSG equation can be written as $[18,19]$.

$\phi(x, X(t))=k \pi-2 \tan ^{-1}\left(\frac{1}{\sqrt{5}} \sinh \left(\sqrt{5} \frac{\sqrt{\lambda}(x-X(t))}{\sqrt{1-\dot{X}^{2}}}\right)\right)$

where $X(t)=x_{0}-\dot{X} t . x_{0}$ and $\dot{X}$ are soliton initial position and its velocity respectively. It is a kink-like solution as figure 1 shows.

We want to investigate the bahaviour of kink solution 3 during the interaction with an external potential. Local external potential $\mathrm{V}(\mathrm{X})$ can be added to the Lagrangian by taking a suitable space dependent function for the parameter $\lambda$ as $\lambda(x)=1+V(x)[2,20,21]$. Therefore the potential can be 


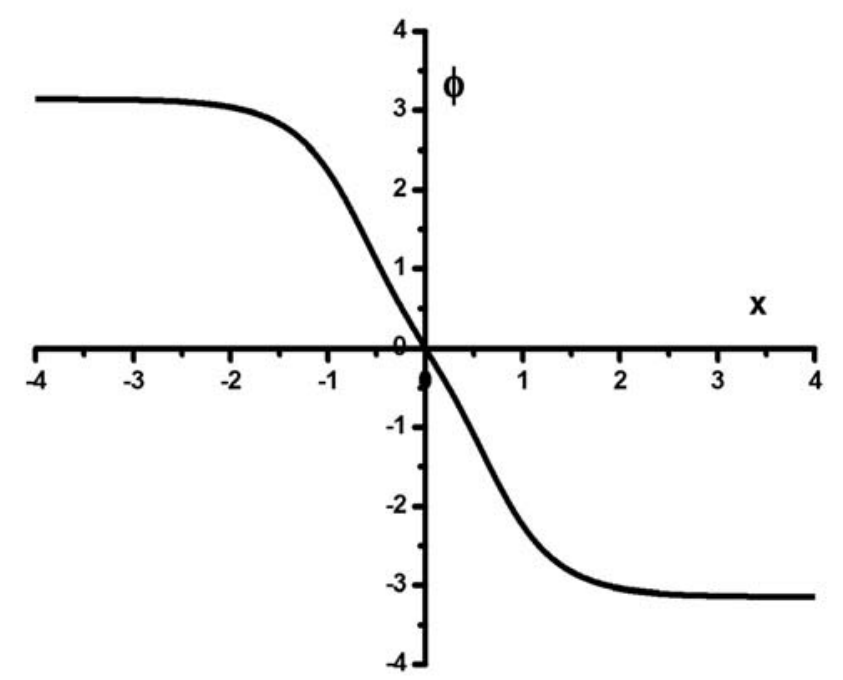

FIG. 1: Kink-like solution of DSG equation (2) as a function of position

found by subtracting usual value of $\lambda=1$ from $\lambda(x)$. Parameter $\lambda(x)$ has been used in [2] as follows

$$
\lambda(x)=\left\{\begin{array}{cc}
1+\lambda_{0} & |x| \leq p \\
1 & |x|>p
\end{array}\right\}
$$

This means that potential $\mathrm{V}(\mathrm{x})$ is a rectangular function with the width of 'p' and the height $\lambda_{0}$. Solution (3) with $\lambda=1$ can be used as initial condition for solving (2) when the potential $\mathrm{V}(\mathrm{x})$ is small.

By inserting the solution (3) (with $\lambda=1$ ) in the Lagrangian (1), using adiabatic approximation [22] we have

$$
\mathcal{L}=\left(\dot{X}^{2}-1-\lambda(x)\right) \frac{50 \cosh ^{2}(\sqrt{5}(x-X(t)))}{\left(5+\sinh ^{2}(\sqrt{5}(x-X(t)))\right)^{2}}
$$

\section{COLLECTIVE COORDINATE VARIABLE}

The center of a soliton can be considered as a particle, if we look at this variable as a collective coordinate. The collective coordinate could be related to the potential by using the Lagrangian (5). This model is able to give us an analytic solution for most of the features of the soliton-potential system. $X(t)$ remains as a collective coordinate if we integrate Lagrangian (5) over the variable $\mathrm{x}$. If we take the potential $V(x)=\varepsilon \delta(x)(\lambda(x)=1+\varepsilon \delta x)$ then (5) becomes

$$
L=\int \mathcal{L} d x=\frac{M_{0}}{2}\left(\dot{X}^{2}-2\right)-\frac{50 \varepsilon \cosh ^{2}(\sqrt{5} X)}{\left(5+\sinh ^{2}(\sqrt{5} X)\right)^{2}}
$$

where

$$
\begin{aligned}
M_{0} & =\int_{-\infty}^{\infty} \frac{100 \cosh ^{2}(\sqrt{5}(x-X(t)))}{\left(5+\sinh ^{2}(\sqrt{5}(x-X(t)))\right)^{2}} d x \\
& =\ln \left(\frac{5+2 \sqrt{5}}{5-2 \sqrt{5}}\right)+4 \sqrt{5}
\end{aligned}
$$

The equation of motion for the variable $\mathrm{X}(\mathrm{t})$ is derived from (6) as

$$
\begin{aligned}
& M_{0} \ddot{X}-100 \varepsilon \sqrt{5} \\
& \times\left(\frac{\cosh (\sqrt{5} X) \sinh (\sqrt{5} X)\left(3-\sinh ^{2}(\sqrt{5} X)\right)}{\left(5+\sinh ^{2}(\sqrt{5} X)\right)^{3}}\right)=0
\end{aligned}
$$

The above equation shows that the peak of the soliton moves under the influence of a complicated force which is a function of external potential $V(x)$ and soliton position. If $\varepsilon>0$ we have a barrier and $\varepsilon<0$ creates a potential well. Figure 2 shows effective force as a function of position for $\varepsilon=0.1$. Fortunately equation (8) has an exact solution as follows

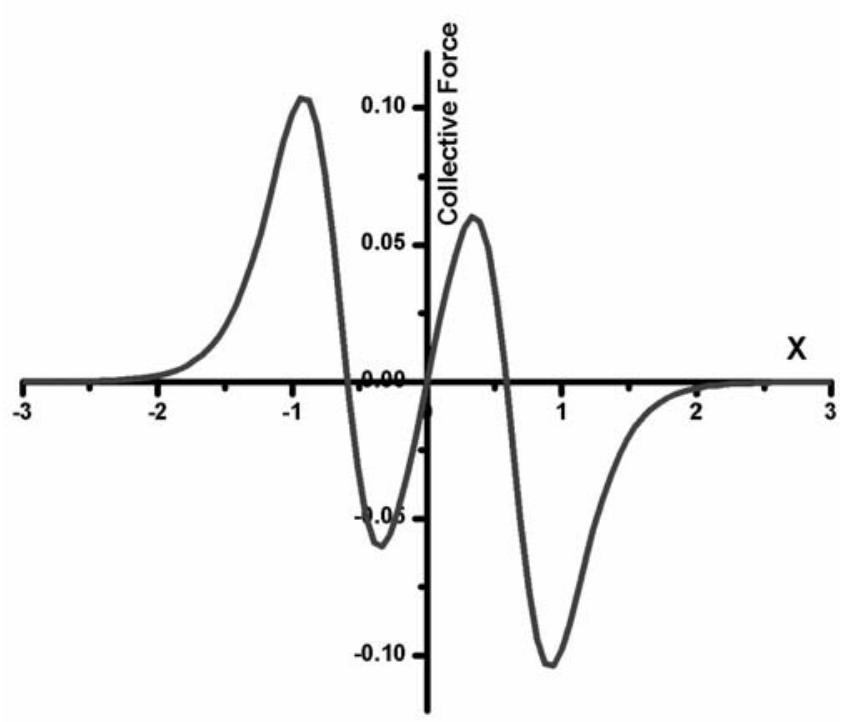

FIG. 2: Effective force acted on the collective particle as a function of position with $\varepsilon=0.1$.

$$
\begin{aligned}
& \frac{1}{2} M_{0}\left(\dot{X}^{2}-\dot{X}_{0}^{2}\right)+50 \varepsilon \\
& \times\left(\frac{\cosh ^{2}(\sqrt{5} X)}{\left(5+\sinh ^{2}(\sqrt{5} X)\right)^{2}}-\frac{\cosh ^{2}\left(\sqrt{5} X_{0}\right)}{\left(5+\sinh ^{2}\left(\sqrt{5} X_{0}\right)\right)^{2}}\right)=0
\end{aligned}
$$

where $X_{0}$ and $\dot{X}_{0}$ are soliton initial position and initial velocity respectively. Collective energy is obtainable from Lagrangian 
(6) as follows

$$
E=\frac{1}{2} M_{0} \dot{X}^{2}+M_{0}+50 \varepsilon \frac{\cosh ^{2}(\sqrt{5} X)}{\left(5+\sinh ^{2}(\sqrt{5} X)\right)^{2}}
$$

It is the energy of a particle with the mass of $M_{0}$ and velocity $\dot{X}$ which is moved under the influence of external effective potential. Therefore $M_{0}$ is indeed the rest mass of one soliton solution (3) of DSG. Figure 3 presents effective potential energy density of a static soliton as a function of its position under the influence of the potential $V(x)=\varepsilon \delta(x)$ with $\varepsilon=0.1$. Thus the effective force is a conservative force and can be described using the effective potential. Equation (10) shows that the effective potential is $E_{P}=50 \varepsilon \frac{\cosh ^{2}(\sqrt{5} X)}{\left(5+\sinh ^{2}(\sqrt{5} X)\right)^{2}}$. From the marginal behaviour of hyperbolic functions at infinity, one can show that the potential decays to zero exponentially when $\mathrm{X}$ goes to infinity. Potential energy has two absolute maxima and one local minimum which can be found by finding the zeros of the effective force. As figure 2 presents, effective force become zero at the origin (center of the external potential) which is the local minimum of the potential.Potential energy reaches its maximum values at $X_{\max }= \pm \frac{\cosh ^{-1}(2)}{\sqrt{(5)}}$. Maximum energy of the soliton is $E_{\max }=\frac{1}{2} M_{0} \dot{X}^{2}+M_{0}+\frac{50 \varepsilon}{16}$. Because of the extended nature of the soliton, the effective potential is not an exact delta function. By substituting $\dot{X}$ from (9) into

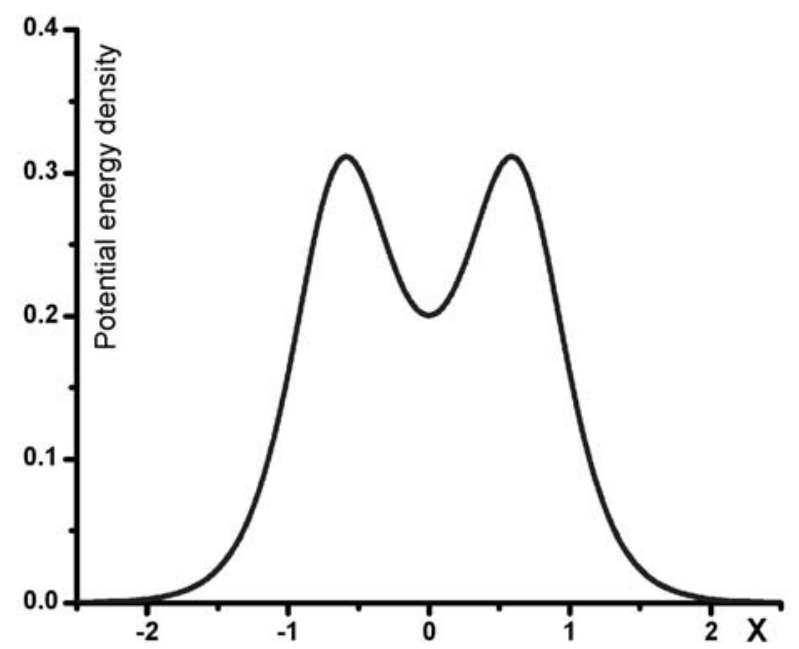

FIG. 3: Collective energy density as a function of position for a static soliton in a delta-like external potential with $\varepsilon=0.1$.

(10) one can show that the energy is a function of soliton initial conditions $X_{0}$ and $\dot{X}_{0}$ only. Therefore the energy of the system is conserved.

Topological charge is [23]

$$
Q=\frac{1}{2 \pi} \int_{+\infty}^{-\infty} \frac{\partial \phi}{\partial X} d X
$$

where $\frac{\partial \phi}{\partial X}$ is topological charge density. For the solution (3) we have

$$
\frac{\partial \phi}{\partial X}=\frac{10 \cosh (\sqrt{5} X)}{5+\sinh ^{2}(\sqrt{5} X)}
$$

Collective topological charge Q can be calculated by Integrating (12) over the variable X. A simple calculation shows that topological charge of one soliton solution is $Q=1$ which is constant and independent of $\mathrm{X}$.

Some features of soliton-potential dynamics can be investigated using equations (9) and (10) analytically which are discussed in the next sections.

\section{POTENTIAL BARRIER}

Suppose that a delta-like potential barrier with the height $\varepsilon$ is located at the origin. There are two different kinds of behaviour for the soliton, during the interaction with the effective potential barrier. It depends on its initial location and its initial velocity. If the soliton is far away from the potential $\left(\left|X_{0}\right|>X_{\max }\right)$ the soliton reflects back or passes over the barrier depends on its initial velocity. Also there is an interesting situation which has not been observed before. If soliton initial position is near to the center of the potential (say $\left|X_{0}\right|<X_{\text {max }}$ ) the soliton is trapped by the barrier and oscillate around the center of the potential, if it has low initial velocity. These situations have been explained in detail in the following.

When the soliton is far from the center of the potential $(X \rightarrow \infty)(10)$ reduces to $E=\frac{1}{2} M_{0} \dot{X}_{0}^{2}+M_{0}$, where $\dot{X}_{0}$ is its initial velocity at infinity. It is the energy of a particle with the rest mass $M_{0}$ and the velocity of $\dot{X}_{0}$. A soliton with a low velocity reflects back from the barrier and a high energy soliton climbs over the barrier and passes through it. So we have a critical value for the velocity of the soliton which separates these two situations. The energy of a soliton at the $X_{\max }$ is $E\left(X=X_{\max }\right)=\frac{1}{2} M_{0} \dot{X}^{2}+M_{0}+\frac{50 \varepsilon}{16}$. The minimum energy for a soliton in this position is $E=M_{0}+\frac{50 \varepsilon}{16}$. On the other hand, a soliton which comes from infinity with initial velocity $v_{c}$ has the energy of $E(X=\infty)=\frac{1}{2} M_{0} v_{c}^{2}+M_{0}$. Therefore the minimum velocity for the soliton to pass the barrier is $v_{c}=\frac{5}{2} \sqrt{\frac{\varepsilon}{M_{0}}}$. The same result is derived by substituting $\dot{X}=0, \dot{X}_{0}=v_{c}, X_{0}=\infty$ and $X=0$ in (10).

If the soliton is located at some position like $X_{0}$ (which is not necessary infinity) the critical velocity will not be $\frac{5}{2} \sqrt{\frac{\varepsilon}{M_{0}}}$. Soliton passes over the barrier if the soliton energy is greater than the energy of a static soliton at $X_{\max }$. So a soliton at the initial position $X_{0}$ with initial velocity $\dot{X}_{0}$ has the critical initial velocity if its velocity becomes zero at $X=X_{\max }$. Consider a soliton with initial conditions of $X_{0}$ and $\dot{X}_{0}$. If we set $X=X_{\max }$ and $\dot{X}=0$ in equation (9) then $v_{c}=\dot{X}_{0}$. Therefore we have

$$
v_{c}=\sqrt{\frac{100 \varepsilon}{M_{0}}\left(\frac{1}{16}-\frac{\cosh ^{2}\left(\sqrt{5} X_{0}\right)}{\left(5+\sinh ^{2}\left(\sqrt{5} X_{0}\right)\right)^{2}}\right)}
$$

Figure 4 shows critical velocity as a function of its initial position. Figure 4 also indicates that solitons with an initial po- 
sition far away from the external potential has critical velocity of $\frac{5}{2} \sqrt{\frac{\varepsilon}{M_{0}}}$.

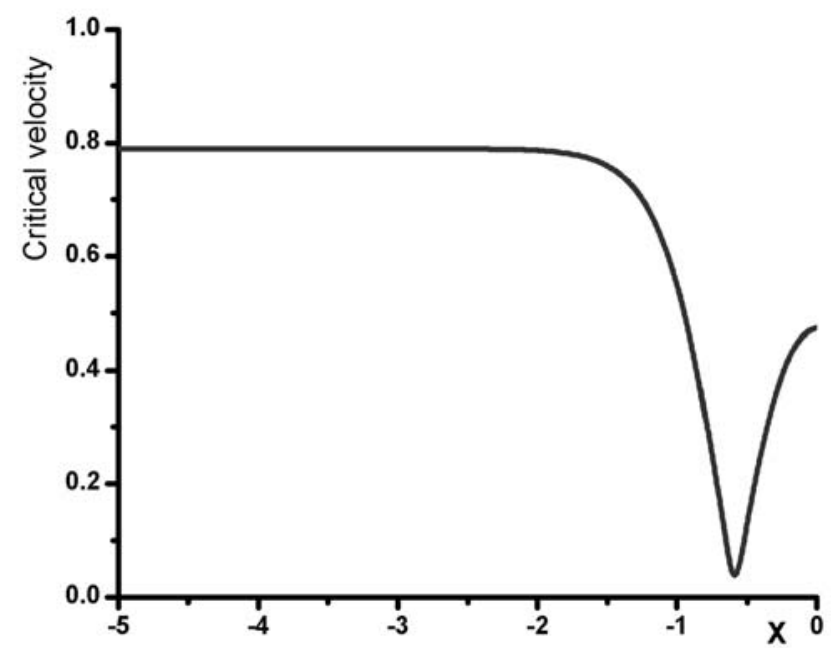

FIG. 4: Critical velocity as a function of initial position $X_{0}$ with $\varepsilon=0.1 M_{0}$.

If soliton at infinity has initial velocity less than the $v_{c}$ then there exists a return point in which the velocity of the soliton is zero. For this situation we have

$$
\frac{\cosh ^{2}\left(\sqrt{5} X_{\text {stop }}\right)}{\left(5+\sinh ^{2}\left(\sqrt{5} X_{\text {stop }}\right)\right)^{2}}=\frac{M_{0}}{100 \varepsilon} \dot{X}_{0}^{2}
$$

Therefore this model predicts linear relation between potential strength $\varepsilon$ and $\dot{X}_{0}^{2}$. Equation (14) shows that complicated term $\frac{\cosh ^{2}\left(\sqrt{5} X_{\text {stop }}\right)}{\left(5+\sinh ^{2}\left(\sqrt{5} X_{\text {stop }}\right)\right)^{2}}$ has linear relation with $\dot{X}_{0}^{2}$ and also $\frac{1}{\varepsilon}$.

A soliton with initial conditions $X_{0}$ and $\dot{X}_{0}$ will go to infinity after the interaction with a potential barrier. The final velocity of the soliton at infinity after the interaction is

$$
\dot{X}=\sqrt{\dot{X}_{0}^{2}+\frac{100 \varepsilon}{M_{0}} \frac{\cosh ^{2}\left(\sqrt{5} X_{0}\right)}{\left(5+\sinh ^{2}\left(\sqrt{5} X_{0}\right)\right)^{2}}}
$$

which is greater than the initial velocity $\dot{X}_{0}$.

Equations (9) and (10) show that the soliton finds its initial velocity after the interaction when it reaches its initial position. This means that the interaction is completely elastic.

\section{SOLITON-WELL SYSTEM}

The soliton-well system is very interesting problem. Suppose a particle moves toward a frictionless potential well. It falls in the well with an increasing velocity and reaches the bottom of the well with its maximum speed. After that, it will climb the well with decreasing velocity and finally pass through the well. Its final velocity after the interaction is equal to its initial speed. But there are some differences between point particle and a soliton in a potential well. Our analytic model explains several features of soliton-well system correctly.

Changing $\varepsilon$ to $-\varepsilon$ in (6) changes potential barrier to potential well. The solution for the system is

$$
\begin{aligned}
& \frac{1}{2} M_{0}\left(\dot{X}^{2}-\dot{X}_{0}^{2}\right)-50 \varepsilon \\
& \times\left(\frac{\cosh ^{2}(\sqrt{5} X)}{\left(5+\sinh ^{2}(\sqrt{5} X)\right)^{2}}-\frac{\cosh ^{2}\left(\sqrt{5} X_{0}\right)}{\left(5+\sinh ^{2}\left(\sqrt{5} X_{0}\right)\right)^{2}}\right)=0
\end{aligned}
$$

There is not a critical velocity for a soliton-well system, but we can define an escape velocity. A soliton with initial position $X_{0}$ reaches the infinity with a zero final velocity if its initial velocity is

$$
\dot{X}_{\text {escape }}=\sqrt{\frac{100 \varepsilon}{M_{0}} \frac{\cosh ^{2}\left(\sqrt{5} X_{0}\right)}{\left(5+\sinh ^{2}\left(\sqrt{5} X_{0}\right)\right)^{2}}}
$$

In other words, a soliton which is located at the initial position $X_{0}$ can escape to infinity if its initial velocity $\dot{X}_{0}$ is greater than the escape velocity $\dot{X}_{\text {escape }}$.

The $X(t)$, trajectory of a soliton during the interaction with the potential well follows from (16) as

$$
t=\int_{X(t=0)}^{X(t)}\left(\sqrt{\dot{X}_{0}^{2}+\frac{100 \varepsilon}{M_{0}} \frac{\cosh ^{2}\left(\sqrt{5} X_{0}\right)}{\left(5+\sinh ^{2}\left(\sqrt{5} X_{0}\right)\right)^{2}}}\right)^{-1} d x
$$

The above integral has been evaluated numerically by using Rumberg's method and X(t) was plotted versus t. This result was compared with direct simulation using equation (2). Figure 4 shows the result for a system with $X_{0}=-5$, $\dot{X}_{0}=-0.1$ and $\varepsilon=-0.2$. There is a little difference between the predicted final velocities from different models after the interaction. The difference is reduced when the height of the potential $(\varepsilon)$ reduces. The difference is due to the approximation which is used for deriving (5) from (1).

Consider a potential well with the depth of $\varepsilon$ and a soliton at the initial position $X_{0}$ which moves toward the well with initial velocity $\dot{X}_{0}$ smaller than the $\dot{X}_{\text {escape }}$. The soliton interacts with the potential and reaches a maximum distance $X_{\max }$ from the center of the potential with a zero velocity and then come back toward the well. The soliton oscillates around the well with the amplitude $X_{\max }$. The required initial velocity to reach $X_{\max }$ is found from (16) as 


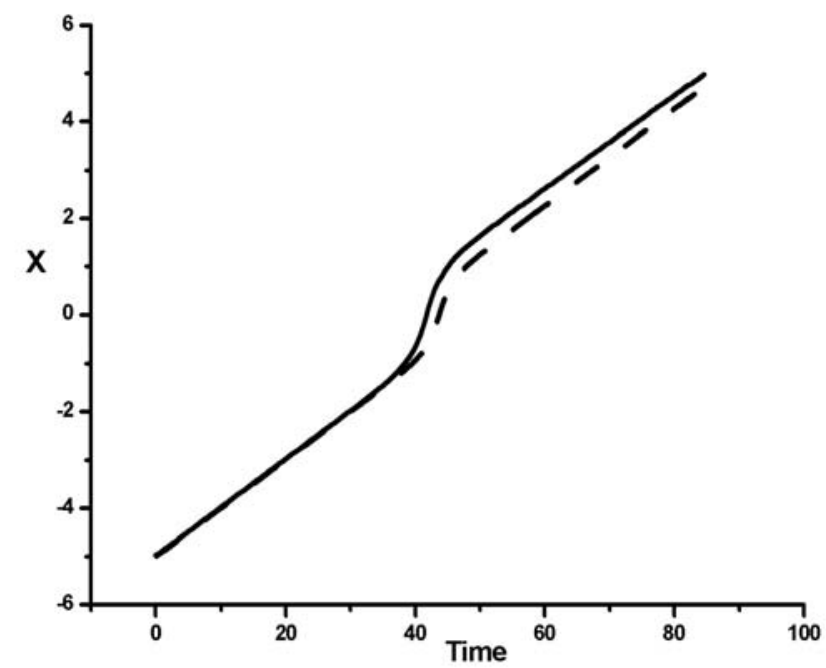

FIG. 5: Soliton trajectory during the interaction with potential well. Potential depth $\varepsilon=-0.2$ has been chosen for direct simulation using (2) (solid line) and $(\varepsilon=-0.134)$ using analytic model (dash line). Initial conditions are $X_{0}=-5$ and $\dot{X}_{0}=0.1$

$$
\dot{X}_{0}=\sqrt{\frac{100 \varepsilon}{M_{0}}\left(\frac{\cosh ^{2}\left(\sqrt{5} X_{0}\right)}{\left(5+\sinh ^{2}\left(\sqrt{5} X_{0}\right)\right)^{2}}-\frac{\cosh ^{2}\left(\sqrt{5} X_{\max }\right)}{\left(5+\sinh ^{2}\left(\sqrt{5} X_{\max }\right)\right)^{2}}\right)}
$$

If the initial velocity is lower than the escape velocity the soliton oscillates around the well. The period of oscillation can be calculated numerically using equation (16).

\section{CONCLUSION AND REMARKS}

An analytical model for the scattering of double sineGordon solitons from delta function potential barriers and also potential wells has been presented. Several features of soliton-potential characters were calculated using this model. A critical velocity for the soliton during the interaction with a potential barrier as a function of its initial conditions and the potential characters has been found. The model predicts specific relations between some functions of initial conditions and other functions of final state of the soliton after the inter- action . An escape velocity has been derived for the solitonwell system. Oscillation period of a soliton in a potential well also has been discussed using this model.

Presented collective coordinate method is able to explain most of the features of double sin-Gordon soliton system during the interaction with an external potential. But this model (like analytical model for single sine-Gordon model in Ref. [16] or presented analytical model for $\phi^{4}$ in Ref.[21]) is not able to explain fine structure of the islands of trapping in soliton-well system. This phenomenon is a very interesting features of soliton-potential systems. It is expected to find an acceptable explanation for this behaviour using a better model with suitable collective coordinate method. On the other hand using this method for investigation of other nonlinear models in potentials is an interesting subject.
[1] Manton N. and Sutcliffe P. 'Topological Solitons' Cambridge university press UK. 2004

[2] Al-Alawi Jassem H. , Zakrzewski W.J. (2007) J. Phys. A 40, 11319

[3] Christova C.V.,Blotza A.,Kim H.C.,Pobylitsaa P.,Watabea T.,Meissnerd Th.,Ruiz Arriolae E. and Goeke K. 1996 Prog.Part.Nucl.Phys. 37

[4] Panico G.,Wulzer A. 2009 Nuclear Physics A 82591114
[5] Biaszak M. 1987 J. Phys. A: Math. Gen. 20 L1253-L1255

[6] Abdalla E.,Maroufia B.,Melgara B. C. and Sedrab M. B. 2001 Physica A 301, 169

[7] McCall S. and Hahn E. L. 1969 Phys. Rev. 183, 457

[8] Frenkel J. and Kontorova T., 1939 J. Phys. [Moscow] 1137

[9] Wysin G., Bishop A. R. and Kumar P.,1984 J. Phys. C 17, 5975

[10] Harold B., 2007 J. High Energy Phys. JHEP03, 055

[11] Fabrizio M.,Gogolin A.O.,Nersesyan A.A., 2000 Nuclear 
Physics B 580 [FS], 647687

[12] Werner P, Refael G and Troyer M 2005 J. Stat. Mech. P12003

[13] Hatakenaka N.,Takayanagi H., Kasai Y. and Tanda S. 2000 Physica B 284-288 563-564

[14] Giuliano1 D. and Sodano P., 2009 arXiv: 0908.0913

[15] Crompton P.R., 2008 arXiv:0810.2087

[16] Javidan K. 2008 PHYS. REV. E 78, 046607

[17] Ghahraman A., Javidan K. 2008 Arxiv:0809.0210

[18] Wazwaz A. 2006 Phys. Lett. A 350367370

[19] Mussardo G., Riva V., Sotkovc G., 2004 Nuclear Physics B 687
[FS] (2004) 189219

[20] Piette B., Zakrzewski W.J. 2007 J. Phys. A: Math. Theor.40 No 2, 329-346

[21] Hakimi E. and Javidan K., 2009 PHYS. REV. E 80, 016606

[22] Kivshar Y. S. , Fei Z. and Vasquez L. 1991 Phys. Rev. Lett 67 1177

[23] Rajaraman R., Solitons and instantons, Elsevier, NorthHolland 1989 